\title{
Schilbach-Rott syndrome associated with 9q22.32q22.33 duplication, involving the PTCH1 gene
}

\author{
Paolo Prontera ${ }^{1}$ Daniela Rogaia ${ }^{1}$ Ester Sallicandro ${ }^{1,2}$ - Amedea Mencarelli ${ }^{1}$ Valentina Imperatore ${ }^{1,2}$. \\ Gabriella Maria Squeo ${ }^{3}$ - Giuseppe Merla $\bigoplus^{3} \cdot$ Sandro Elisei $^{4} \cdot$ Danilo Moretti-Ferreira $^{5}$ - Susanna Esposito ${ }^{6}$. \\ Gabriela Stangoni ${ }^{1}$
}

Received: 27 April 2018 / Revised: 5 March 2019 / Accepted: 12 March 2019 / Published online: 1 April 2019

(c) European Society of Human Genetics 2019

\begin{abstract}
Schilbach-Rott syndrome (SRS, OMIM\%164220) is a disorder of unknown aetiology that is characterised by hypotelorism, epichantal folds, cleft palate, dysmorphic face, hypospadia in males and mild mental retardation in some patients. To date, 5 families and 17 patients have exhibited this phenotype, and recurrence in two of these families suggests an autosomal dominant inheritance. SRS overlaps with a mild form of holoprosencephaly (HPE), but array-CGH analysis and sequencing of some HPE-related genes (SEPT9, SHH and TWIST) did not reveal any variants in at least one family. Herein, we investigated by array-CGH analysis a 11-year-old female patient and her father, both exhibiting the typical SRS phenotype, disclosing in the daughter-father couple the same microduplication of chromosome 9q22.32q22.33 [arr[hg19]9q22.32 $\left.\left(98,049,611 \_98,049,636\right) \times 3,9 q 22.33\left(99,301,483 \_99,301,508\right) \times 3\right]$, involving eight genes, including PTCH1. The duplication segregated with the disease, since it was not found in the healthy paternal grandparents of the proband. The gain-offunction variants of the PTCH1 gene are responsible for a mild form of HPE. This is the first genetic variant found in SRS. This finding reinforces the hypothesis that SRS belongs to the HPE clinical spectrum and suggests to perform array-CGH in patients with SRS phenotype and, if negative, to consider a potential benefit from sequencing of HPE-related genes.
\end{abstract}

\section{Introduction}

Schilbach and Rott [1] described ten relatives over five generations who exhibited a congenital disorder that is characterised by ocular hypotelorism, submucosal cleft

Paolo Prontera

paolo.prontera@ospedale.perugia.it

1 Medical Genetics Unit, University-Hospital "Santa Maria della Misericordia", Perugia, Italy

2 "Mauro Baschirotto" Institute for Rare Diseases (B.I.R.D.), Via B. Bizio, 1, Costozza di Longare, 36023 Costozza di Longare, VI, Italy

3 Division of Medical Genetics, IRCCS Casa Sollievo della Sofferenza, San Giovanni Rotondo, Foggia, Italy

4 Istituto Serafico, Assisi, Perugia, Italy

5 Department of Genetics, Institute of Biosciense of Botucatu, São Paulo State University - UNESP, São Paulo, Brazil

6 Pediatric Clinic, Department of Surgical and Biomedical Sciences, University of Perugia, Perugia, Italy palate and hypospadias in males. Other anomalies included blepharophimosis, upslant of palpebral fissures and a tendency for cutaneous syndactyly of the 3rd and 4th fingers and 2nd and 3rd toes. In 2002, Joss et al. [2] described a mother and two sons with cleft palates and facial appearances that closely resembled the Schilbach-Rott syndrome (SRS) (OMIM\%164220). A father-son pair from Mexico who exhibited the typical features of SRS, such as ocular hypotelorism, cleft palate, hypospadias (only in the child) and microcephaly, further supports an autosomal inheritance [3]. De Carvalho et al. [4] described a 4-year-old girl with blepharophimosis, which is a typical facial gestalt and skeletal abnormalities seen in the blepharofacioskeletal syndrome (BFSS), and found a clinical overlap with SRS; the findings suggested that BFSS and SRS were of the same condition. Shkalim et al. [5] described another family with an autosomal dominant syndrome of hypotelorism, cleft palate/uvula, high-arched palate and mild mental retardation - symptoms resembling SRS - and noted a similarity in the facial appearance of patients with holoprosencephaly, even in the absence of holoprosencephaly on MRI. The results from genetic studies were normal in this case, including 
FISH for deletion of 22q11, karyotype analysis, fragile $\mathrm{X}$ testing, high-resolution comparative genomic hybridisation (CGH) and SEPT9, SHH and TWIST sequence analyses. These data suggest that SRS is an autosomal dominant developmental disorder of unknown aetiology.

Herein, we described a father-daughter couple exhibiting an SRS phenotype in whom we identified a 9q22.32q22.33 microduplication involving the $P T C H 1$ gene.

\section{Materials and methods}

Informed consent for the genetic studies and the publication of images was obtained from all family members. Cognitive evaluations of patients were performed using the Wechsler Intelligence Scale for Children, revision III (WISC-III). Array-CGH analyses were performed on DNA extracted from peripheral blood of the proband and her parents using the Agilent Human Genome CGH Microarray Kit $60 \mathrm{~K}$ (Agilent Technologies, Santa Clara, California, USA), as described previously [6, 7]. The array-CGH results were validated by a second array-CGH analysis. DNA extracted from peripheral blood was also obtained for paternal grandparents of the proband and a real-time PCR (QPCR) on the $P T C H 1$ gene was performed, as previously described [8].

\section{Subjects}

The patient, evaluated 2 years ago, was a 11-year-old female, the only child of a non-consanguineous couple. The personal and family history of her mother and family history of her father were unremarkable. The personal history of the father was characterised by the presence of anterior glandular hypospadias at birth, which was surgically treated at 18 months of age. He exhibited dysmorphisms of the face very similar to the daughter, including microbrachicephaly, hypotelorism, flat face, short philtrum, beaked and long nose with a notch on the tip, high-arched palate, short palpebral fissures, blepharophimosis and clinodactyly of 5th fingers (Fig. 1a, b). The paternal grandparents of the proband were healthy and did not show dysmorphism of the face. The proband's pregnancy was complicated by maternal bleeding during the first trimester. Foetal ultrasound performed at 22 weeks of gestation revealed a foetal central cleft lip and a deceleration of foetal growth was observed during the last month of gestation. The parents decided to not perform invasive prenatal genetic testing. Spontaneous vaginal delivery occurred at 40 weeks of gestation, with a birth weight of $2300 \mathrm{~g}$ ( $<3 \mathrm{rd}$ percentile), a length of $47 \mathrm{~cm}$ (10th-25th percentile) and an OFC of $31 \mathrm{~cm}$ (3rd percentile). A central cleft of the lip and palate was immediately evident and surgically treated during the first few months of life. Motor development was achieved normally, but speech was delayed. Therefore, she received speech therapy. The patient did not experience seizures or other neurological disorders. Diagnostic workup included haematological and biochemical testing, urinalysis, ECG and echocardiography, ABR, abdominal ultrasounds, including urinary system evaluation and brain MRI, all of which were unremarkable. Her height was $140 \mathrm{~cm}$ (25th centile), her weight was $35.5 \mathrm{~kg}$ (50th centile) and her OFC was $48.5 \mathrm{~cm}(<<3 \mathrm{rd}$ centile) at the time of our evaluation (11 years old). Physical examination disclosed microbrachicephaly, short palpebral fissures, blepharophimosis, hypotelorism, long nose, short philtrum, flat malar region, asymmetric face with ptosis of the right eye, dental crowding, frontal angioma and clinodactyly of the 5th fingers (Fig. 1c, d).

\section{Results}

The proband evidenced a full-scale IQ of 70, performance IQ of 75 and a verbal IQ of 65 on the WISC-III, which is in the borderline intellectual functioning range. The array-CGH analysis of the proband revealed a $1.2-\mathrm{Mb}$ duplication of chromosome 9q22.32q22.33 [arr[hg19] 9q22.32(98,049,611_98,049,636)x3,9q22.33 (99,301,483_ $99,301,508) \times 3]$. These two patients have been registered in the Decipher database (https://decipher.sanger.ac.uk/) with the following identification codes: SAN376908 (proband) and SAN376909 (father) [9]. The array-CGH analyses were extended to the parents and disclosed the paternal origin of the duplication. These results were confirmed in the proband and in her father using a second array-CGH analysis (Fig. 2). We also performed a QPCR analysis of the PTCH1 gene in the healthy paternal grandparents who did not show the $9 \mathrm{q} 22.32 \mathrm{q} 22.33$ microduplication (Fig. 3). The rearrangement involved the following OMIM genes: $F A N C C$, PTCH1, HSD17B3, ERCC6L2, SLC35D2, HABP4, ZNF367 and CDC14B (Fig. 2).

\section{Discussion}

SRS is a very rare dysmorphic condition of unknown aetiology, with less than 20 patients reported to date [1-5]. SRS patients share facial features with patients who have holoprosencephaly (HPE), even when their brain appears normal, as in the microform of HPE [5, 10]. Microforms are defined as HPE with facial dysmorphisms (ocular hypothelorism, midface hypoplasia, cleft lip and/or palate), minimal (corpus callosum defect, posterior fossa abnormalities) or no brain involvement, possible extra craniofacial congenital defects, mostly visceral and renal/urinary anomalies and intellectual disability of various degrees [5]. Shkalim et al. [5] analysed the $S H H$ gene, which is the gene 
Fig. 1 a, b Father of the proband, 40 years old. Note the hypotelorism, short philtrum, beaked, long nose with a notched tip, posteriorly rotated ears, short palpebral fissures and asymmetric face with ptosis of the left eye. c, d Proband, 11 years old. Note the similarity with the paternal phenotype: hypotelorism, short philtrum, long nose, small mouth with surgical scar of labiopalatoschisis, flat midface, posteriorly rotated ears, short palpebral fissures and asymmetric face with ptosis of the left eye

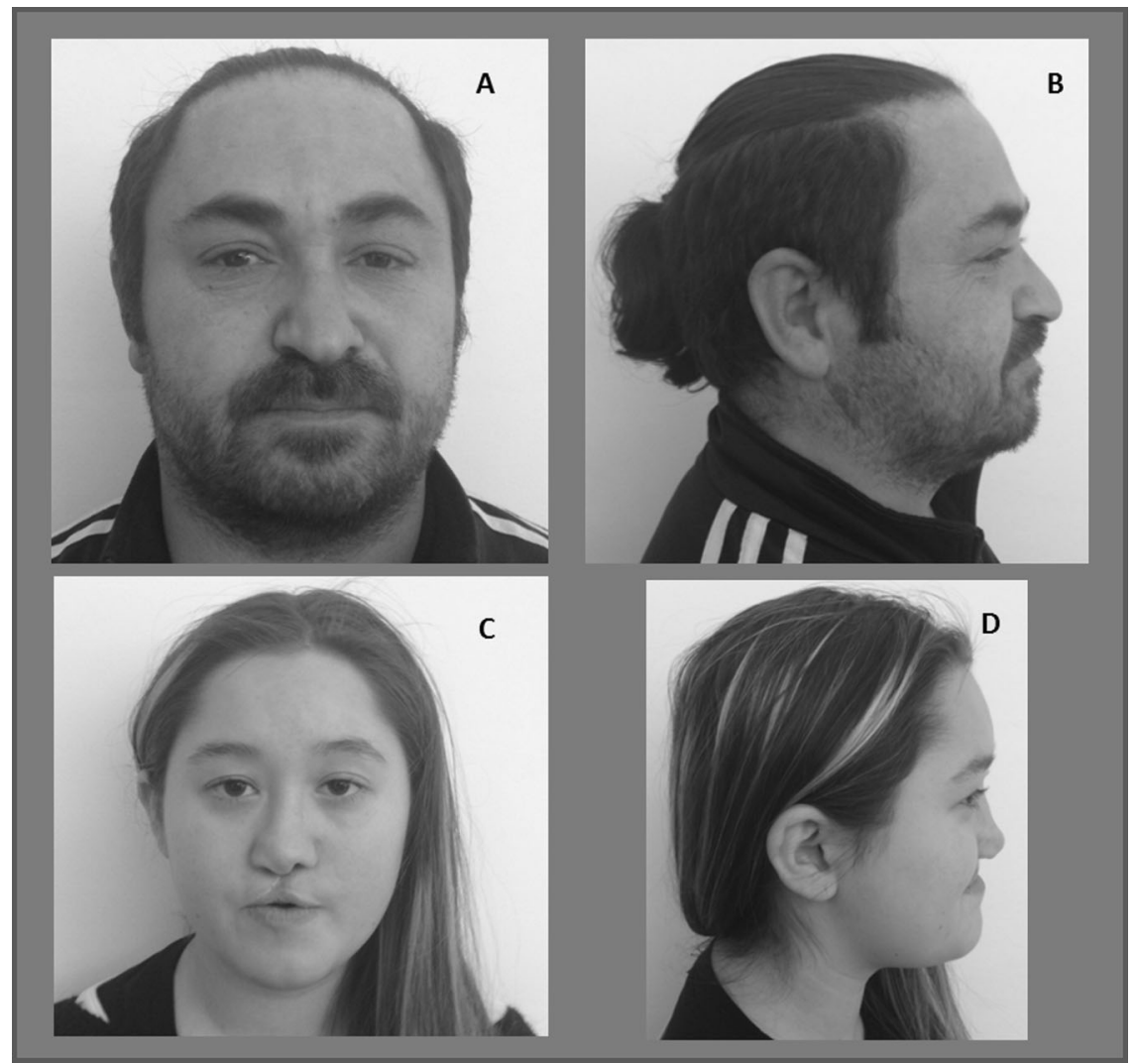

most frequently involved in HPE in SRS patients; however, no functionally relevant variants were found. However, HPE exhibits a wide genetic heterogeneity with at least 12 different loci and 7 identified genes (SHH, SIX3, PTCH1, GLI2, ZIC2, CDON and TGIF) [11]. SHH is also the gene most commonly responsible for HPE microform [10]. Notably, in contrast to the loss-of-function variants and genomic deletions of PTCH1 found in basal cell naevus syndrome (BCNS or Gorlin syndrome, OMIM \#109400), at least five different missense PTCHI variants, encoding for the $\mathrm{SHH}$ receptor, that normally acts to repress $\mathrm{SHH}$ signalling [11], were found in patients with HPE or holoprosencephaly-like features and normal brain (HPE7 or HPE microform) [12-14]. These variants likely affect the protein function through a gain-of-function manner [14]. Moreover, Derwinska et al. [15] identified an $\sim 360-\mathrm{kb}$ duplication in 9q22.32, involving the entire PTCH1 gene, in a 21-month-old boy and his mother, who both exhibited a mild intellectual disability and microcephaly. Izumi et al. [16] described a syndrome that was characterised by short stature, microcephaly, cognitive delay and facial features in three patients (mother and two children) carrying a microduplication of $\sim 2.3 \mathrm{Mb}$ completely involving the PTCH1 locus. Other larger duplications involving the $9 q 22 q 31$ were described in at least 30 patients who shared the following clinical features: short stature, microcephaly, intellectual disability, dysmorphic facial features and congenital heart disease [17-19]. However, there were small duplications of 9q22.32, such as those described by Derwinska et al. [15]. Izumi et al. [17] in this report, restrict the number of genes that are potentially responsible for the primary clinical phenotype of this newly, clinically recognisable, duplication syndrome (Fig. 4). In Table 1, we compare the phenotypes of SRS with those of our patients, patients with a small 9q22.3 microduplication and patients with gain-of-function PTCH1 variants. There are many clinical features shared by these patients, such as microcephaly, short stature, high or cleft palate, hypotelorism, syndactyly or clinodactyly, and usually mild, intellectual disability; instead, other characteristics, such as brain anomalies and hypospadia, are more variable.

The role of PTCHI in the pathogenesis of the main features (microcephaly, hypotelorism, high palate and intellectual disability) that characterise the phenotype associated with 9q22.3 microduplication appears likely. In fact, it is plausible that a duplication of the entire PTCH1 gene, which might lead to an overexpression of the protein, could reduce $\mathrm{SHH}$ signalling during embryogenesis in a dose-dependent manner, since PTCH1 physiologically suppresses SHH activity. The expected clinical effect of SHH signalling repression is HPE, which is similar to the phenotype of patients with loss-of-function $\mathrm{SHH}$ variants. 
Fig. 2 In the upper figure, the three partial array-CGH profiles of the mother, proband and father show the presence of microduplication in the last two subjects involving the chromosomal region 9q22.32q22.33. In the lower figure, the gene content is illustrated. Please note the presence of the entire $\mathrm{PTCHI}$ gene

Fig. 3 Results of the QPCR performed on exons (ex) 6, 14, 23 of the PTCH1 gene (genomic DNA) in two healthy controls CTRL_A (grey) and CTRL_B (black), paternal grandmother (blue), paternal grandfather (red), proband (green) and her father (purple). Note that duplication of $P T C H 1$ is present only in the father-daughter couple
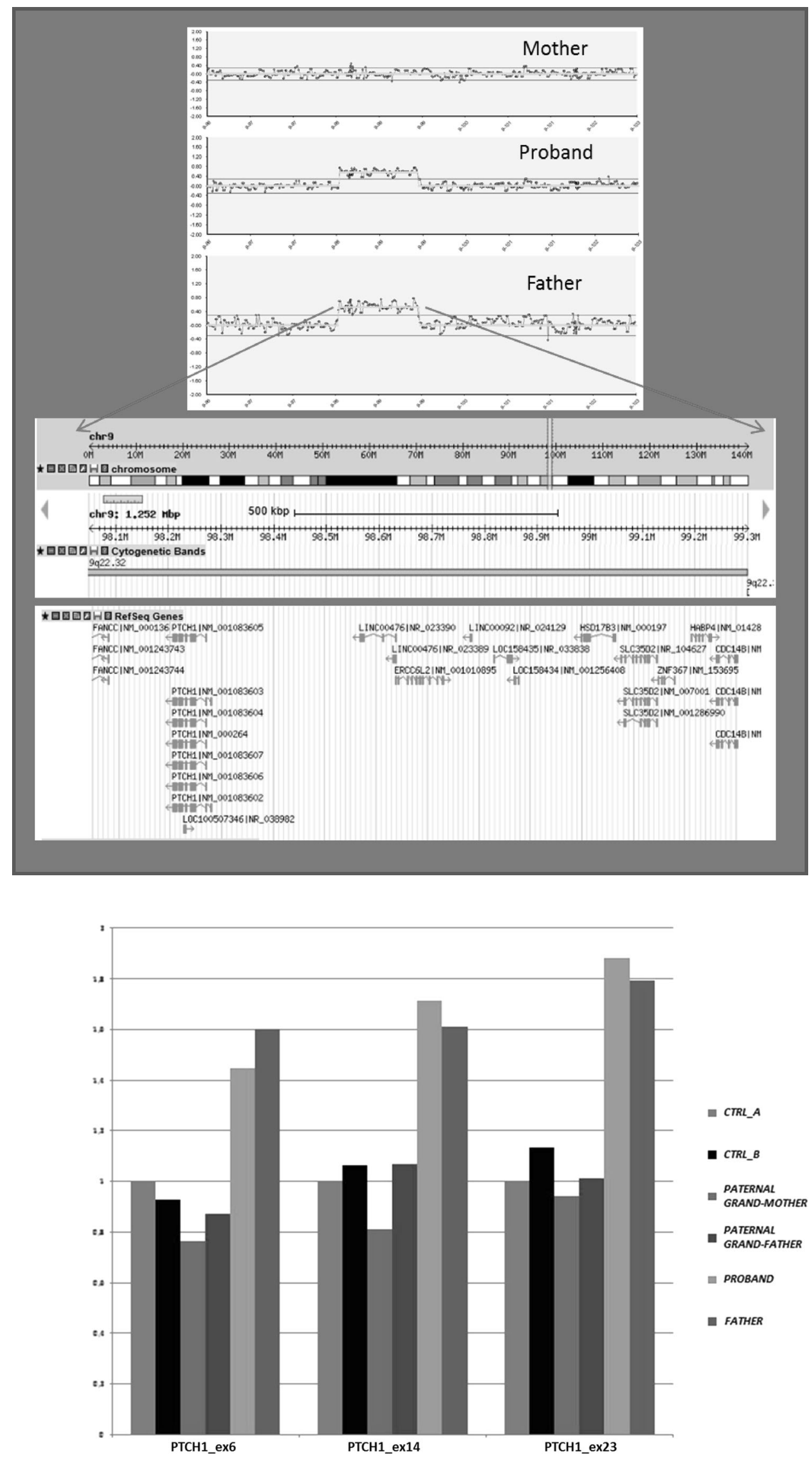


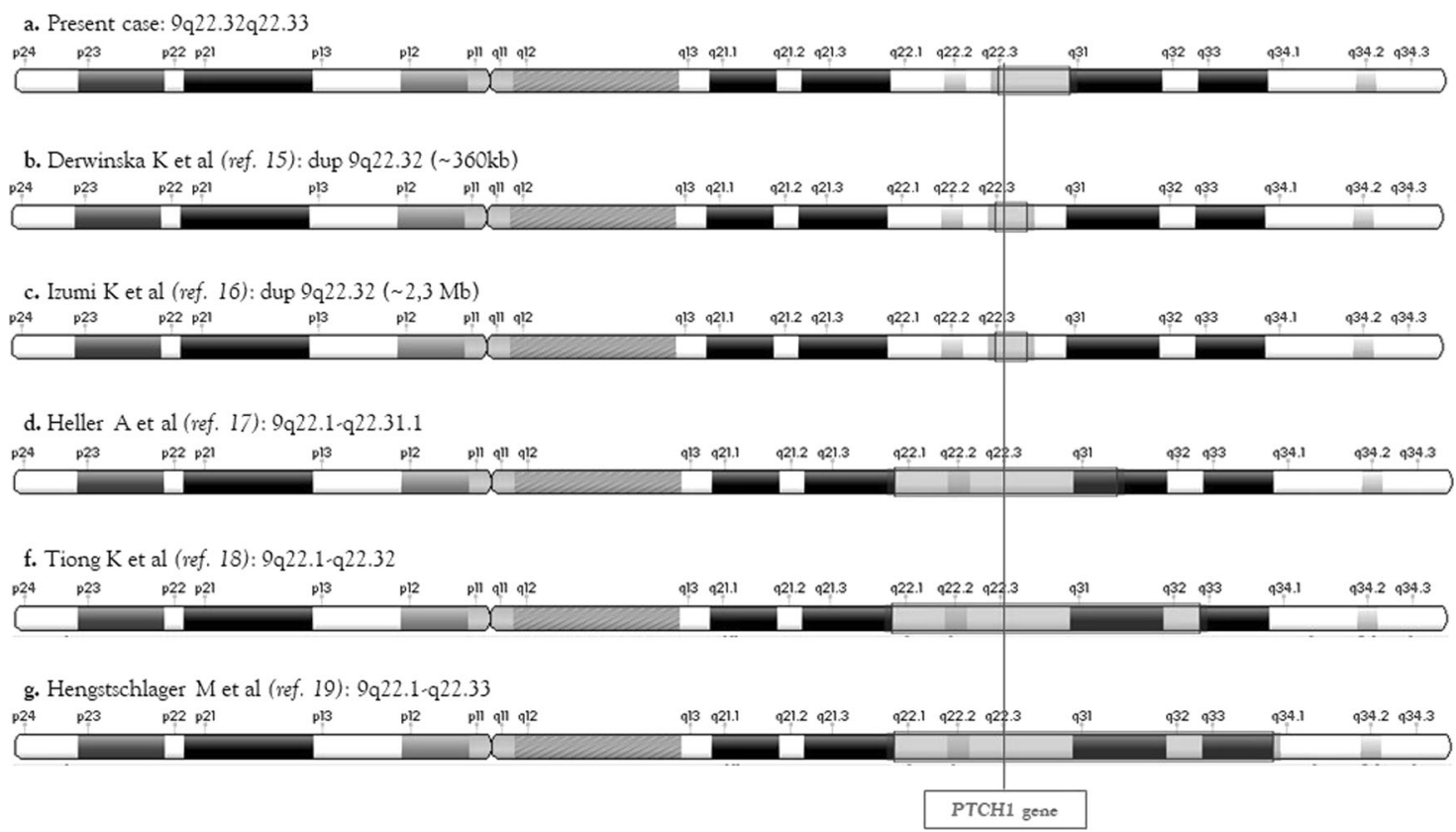

Fig. 4 Summary of published reports of chromosome 9q duplication (blue rectangles), partially overlapping to ours. The position of PTCH1 is highlighted by the red line. Note that this gene is also involved in smaller duplications

We used RT-PCR to attempt to dose RNA levels of PTCH1 in the peripheral blood cells of our patients, in order to verify whether the $P T C H 1$ gene duplication produces a higher $\mathrm{PTCH} 1$ transcript expression. However, this gene is physiologically expressed at extremely low levels in this tissue, and these experiments failed (data not shown). The proband and her father declined the request to perform a skin biopsy that would be used to obtain fibroblasts, cells where $\mathrm{PTCHI}$ expression is more pronounced. If the role of PTCH1 appears plausible in contributing to the SRS phenotype, the role of the microduplication $9 \mathrm{q} 22.32 \mathrm{q} 22.33$ appears more obvious, since it segregates with the disease, being present in the affected father-daughter pair but absent in the healthy paternal grandparents of the proband. We cannot exclude a possible clinical contribution of other duplicated genes and non-coding RNA in the $9 q 22.32 q 22.33$ region, but, to date, none of these genes has known to be implicated in HPE and/or autosomal dominant conditions. Biallelic loss-of-function FANCC variants are responsible for classical Fanconi anaemia (OMIM \#227645); biallelic loss-of-function HSD17B3 variants are responsible for 17-beta-hydroxysteroido dehydrogenase III deficiency, which is an autosomal recessive form of male pseudohermaphrodistism (OMIM \#264300); biallelic lossof-function ERCC6L2 variants are responsible for bone marrow failure syndrome 2 (OMIM \#615715). Until now, the other duplicated genes (SLC35D2, ZNF367, HABP4 and $C D C 14 B)$ are not known to be associated with human diseases.
The presence of hypospadias in our patient and in SRS merits a final comment. SHH is one of the most important signalling molecules that contributes to genital tuberculum (GT) outgrowth and differentiation. SHH is expressed in the urethral plate epithelium (UE) during the hormoneindependent phase of genital male embryogenesis, and it activates its pathway via the PTCH receptor, which is required for outgrowth, patterning and cell survival in the developing GT [20]. Mice with a targeted deletion of Shh exhibit initiation of the genital swellings, but outgrowth is not maintained, which indicates the absence of external genitalia [21]. Moreover, in a large Californian cohort of patients with hypospadias, several SNPs in SHH were associated with an increased risk [22]. Taken together, these observations suggest that hypospadias in our patient might result from the same SHH signalling dysfunction that leads to HPE microforms.

In summary, we identified a 9q22.32q22.33 duplication involving the $\mathrm{PTCH} 1$ gene in a father-daughter couple with SRS. This "copy number variation" is the first genetic variant found in SRS, and since array-CGH was negative in another SRS patient, genetic heterogeneity seems plausible. This finding supports the autosomal dominant inheritance of SRS and suggests that this condition belongs to the HPEmicroform subgroup. These preliminary data suggest to perform array-CGH analysis and sequencing of HPErelated genes in the presence of an SRS phenotype. Further studies in a larger cohort of SRS patients are needed to reach a definitive conclusion. 
Table 1 Clinical comparison among SRS patients described here, other patients with a small overlapping 9q22.3 microduplication and patients with $P T C H 1$ gain-of-function variants

\begin{tabular}{|c|c|c|c|c|}
\hline Features & SRS & $\begin{array}{l}\text { Present } \\
\text { patients }\end{array}$ & $\begin{array}{l}\text { Patients with a small } \\
\text { overlapping 9q22.3 }_{\text {duplication }^{\mathrm{a}}}\end{array}$ & $\begin{array}{l}\text { Patients with } \mathrm{GoF} \\
P T C H 1 \text { variants }\end{array}$ \\
\hline $\begin{array}{l}\text { Autosomal dominant } \\
\text { inheritance }\end{array}$ & + & + & + & + \\
\hline Short stature & $+l-$ & $+/-$ & + & + \\
\hline Microcephaly & + & + & + & + \\
\hline Asymmetric face & + & + & + & + \\
\hline Micrognathia & + & - & + & + \\
\hline $\begin{array}{l}\text { Posteriorly rotated ears/ } \\
\text { hypoplastic ears }\end{array}$ & + & + & - & - \\
\hline Hypotelorism & + & + & $+1-$ & $+1-$ \\
\hline Upslanting palpebral fissures & + & - & + & + \\
\hline Blepharophimosis & + & + & - & - \\
\hline Epicanthal folds & + & - & + & + \\
\hline Long nose, prominent nose & + & + & + & + \\
\hline $\begin{array}{l}\text { High or cleft palate, bifid } \\
\text { uvula }\end{array}$ & + & + & + & + \\
\hline Small mouth & + & + & - & - \\
\hline Hypospadias (male) & + & + & - & - \\
\hline Syndactyly, clinodactyly & + & + & + & + \\
\hline Mental retardation & Mild & Mild & Mild & Mild \\
\hline HPE & - & - & - & $+1-$ \\
\hline
\end{tabular}

SRS Schilbach-Rott syndrome, HPE holoprosencephaly, + feature present, - feature absent, +/- feature present only in some patients

${ }^{a}$ Patients reported by Derwinska et al. [15] and Izumi et al. [16]

${ }^{\mathrm{b}}$ Patients reported by Ming et al. [13] and Ribeiro et al. [14]
Acknowledgements We gratefully acknowledge the family who participated in this study. This research was supported by the "Mauro Baschirotto Institute for Rare Diseases" Foundation. The funders played no role in the design of the study, the data collection, analysis and interpretation or writing of the paper.

\section{Compliance with ethical standards}

Conflict of interest Dr. Sallicandro's work has been funded by the "Baschirotto Institute of Rare Disease" (BIRD) Foundation. She has received compensation for carrying out a study on possible genetic causes of Schilbach-Rott syndrome. All other authors declare that they have no conflict of interest.

Publisher's note: Springer Nature remains neutral with regard to jurisdictional claims in published maps and institutional affiliations.

\section{References}

1. Schilbach U, Rott HD. Ocular hypotelorism, submucosal cleft palate, and hypospadias: a new autosomal dominant syndrome. Am J Med Genet. 1988;31:863-70.

2. Joss SK, Paterson W, Donaldson MDC, Tolmie JL. Cleft palate, hypotelorism, and hypospadias: Schilbach-Rott syndrome. Am J Med Genet. 2002;113:105-7.
3. Becerra-Solano LE, Casillas-Avila MP, Diaz-Rodriguez M, Nastasi-Catanese JA, Toscano-Flores JJ, Ramirez-Duenas ML. Schilbach-Rott syndrome in a third family: further delineation of an autosomal dominant trait. Genet Couns. 2007;18:317-23.

4. de Carvalho DR, Rossi NF, Schellini S, Moretti-Ferreira D, Richieri-Costa A. Schilbach-Rott/blepharofacio skeletal syndrome in a Brazilian patient. Am J MedGenet. 2008;146A:2134-7.

5. Shkalim V, Baris HN, Gal G, Gleiss R, Calderon S, Wessels M, et al. Autosomal dominant syndrome of mental retardation, hypotelorism, and cleft palate resembling Schilbach-Rott syndrome. Am J Med Genet. 2009;149A:2700-5.

6. Prontera P, Bernardini L, Stangoni G, Capalbo A, Rogaia D, Romani R, et al. Deletion 2p15-16.1 syndrome: case report and review. Am J Med Genet. 2011;155A:2473-8.

7. Tokita MJ, Chow PM, Mirzaa G, Dikow N, Maas B, Isidor B, et al. Five children with deletions of $1 \mathrm{p} 34.3$ encompassing AGO1 and AGO3. Eur J Hum Genet. 2015;23:761-5.

8. Howald C, Merla G, Digilio MC, Amenta S, Lyle R, Deutsch S, et al. Two high throughput technologies to detect segmental aneuploidies identify new Williams-Beuren syndrome patients with atypical deletions. J Med Genet. 2006;43:266-73.

9. Firth HV, et al. DECIPHER: database of chromosomal imbalance and phenotype in humans using ensembl resources. Am J Hum Genet. 2009;84:524-33.

10. Mercier S, Dubourg C, Garcelon N, Campillo-Gimenez B, Gicquel I, Belleguic M, et al. New findings for phenotype-genotype correlations in a large European series of holoprosencephaly cases. J Med Genet. 2011;48:752-60. 
11. Wallis D, Muenke M. Mutations in holoprosencephaly. Hum Mutat. 2000;16:99-108.

12. Marigo V, Davey RA, Zuo Y, Cunningham JM, Tabin CJ. Biochemical evidence that patched is the Hedgehog receptor. Nature. 1996;384:176-9.

13. Ming JE, Kaupas ME, Roessler E, Brunner HG, Golabi M, Tekin M, et al. Mutations in PATCHED-1, the receptor for SONIC HEDGEHOG, are associated with holoprosencephaly. Hum Genet. 2002;110:297-301.

14. Ribeiro LA, Murray JC, Richieri-Costa A. PTCH mutations in four Brazilian patients with holoprosencephaly and in one with holoprosencephaly-like features and normal MRI. Am J Med Genet A. 2006;140:2584-6.

15. Derwińska K, Smyk M, Cooper ML, Bader P, Cheung SW, Stankiewicz P. PTCH1 duplication in a family with microcephaly and mild developmental delay. Eur J Hum Genet. 2009;17:267-71.

16. Izumi K, Hahn A, Christ L, Curtis C, Neilson DE. Familial 9q22.3 microduplication spanning PTCH1 causes short stature syndrome with mild intellectual disability and dysmorphic features. Am J Med Genet A. 2011;155:1384-9.
17. Heller A, Seidel J, Hübler A, Starke H, Beensen V, Senger G, et al. Molecular cytogenetic characterisation of partial trisomy $9 \mathrm{q}$ in a case with pyloric stenosis and a review. J Med Genet. 2000;37:529-32.

18. Tiong K, Cotterill A, Falhammar H. Adult case of partial trisomy 9q. BMC Med Genet. 2010;11:26.

19. Hengstschläger M, Prusa AR, Repa C, Drahonsky R, Deutinger J, Pollak A, et al. Patient with partial trisomy $9 \mathrm{q}$ and learning disability but no pyloric stenosis. Dev Med Child Neurol. 2004;46: $57-59$.

20. Bouty A, Ayers KL, Pask A, Heloury Y, Sinclair AH. The genetic and environmental factors underlying hypospadias. Sex Dev. 2015;9:239-59.

21. Haraguchi R, Mo R, Hui C, Motoyama J, Makino S, Shiroishi T, et al. Unique functions of Sonic hedgehog signaling during external genitalia. Development. 2001;128:4241-50.

22. Carmichael SL, Ma C, Choudhry S, Lammer EJ, Witte JS, Shaw GM. Hypospadias and genes related to genital tubercle and early urethral development. J Urol. 2013;190:1884-92. 